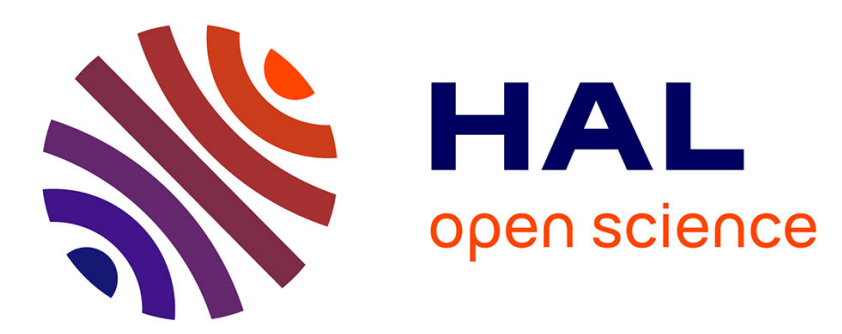

\title{
Towards Cognitive Radio Networks: Spectrum Utilization Measurements in Suburb Environment
}

Vaclav Valenta, Zbynek Fedra, Roman Marsalek, Geneviève Baudoin, Martine Villegas

\section{- To cite this version:}

Vaclav Valenta, Zbynek Fedra, Roman Marsalek, Geneviève Baudoin, Martine Villegas. Towards Cognitive Radio Networks: Spectrum Utilization Measurements in Suburb Environment. Radio and Wireless Symposium, Jan 2009, United States. pp.ISBN: 978-1-4244-2699- 7. hal-00446958

\section{HAL Id: hal-00446958 https://hal.science/hal-00446958}

Submitted on 13 Jan 2012

HAL is a multi-disciplinary open access archive for the deposit and dissemination of scientific research documents, whether they are published or not. The documents may come from teaching and research institutions in France or abroad, or from public or private research centers.
L'archive ouverte pluridisciplinaire HAL, est destinée au dépôt et à la diffusion de documents scientifiques de niveau recherche, publiés ou non, émanant des établissements d'enseignement et de recherche français ou étrangers, des laboratoires publics ou privés. 


\title{
Towards Cognitive Radio Networks: Spectrum Utilization Measurements in Suburb Environment
}

\author{
Václav Valenta ${ }^{1,2}$, Zbynek Fedra' ${ }^{1}$, Roman Maršálek ${ }^{1}$, Geneviève Baudoin ${ }^{2}$, Martine Villegas ${ }^{2}$ \\ ${ }^{1}$ Dept. of Radio Electronics, Brno University of Technology, Brno, 62100, Czech Republic \\ ${ }^{2}$ Université Paris-Est, ESYCOM, ESIEE, Cité Descartes, 93162 Noisy-le-Grand, France
}

\begin{abstract}
This paper deals with spectrum utilization measurements in the frequency band from $100 \mathrm{MHz}$ up to $3 \mathrm{GHz}$. The measurement is based on the energy detection principle using wideband logarithmically periodic antenna. The results point out the fact, that the frequency spectrum is not utilized in an optimal manner and that there do exist less or more utilized licensed frequency bands that could be possibly used by cognitive radios in an opportunistic way. Cognitive radio concept for better spectrum utilization is introduced here along with an overall approach regarding spectrum utilization in the next generation wireless networks.
\end{abstract}

Index Terms - Cognitive radio, radio spectrum management, spectrum sensing, spectrum utilization.

\section{INTRODUCTION}

The electromagnetic spectrum is a scarce natural resource and as any other finite resource, it is limited by its usability. Therefore, the electromagnetic spectrum has to be regulated by relevant governmental authorities (ITU, CTU, FCC etc). Actual wireless communication systems are mainly based on fixed resource allocation. The radio spectrum is assigned or sold to users on long term basis, covering large regions like whole countries. However, as many measurement studies reveal [1], [2], this approach to the frequency allocation leads into vast underutilization of frequency spectrum due to very sporadic usage within various geographical regions as well as over a given period of time. These results lead us to reconsider the allocation protocol of the frequency spectrum and moreover, it opens space for novel and more efficient wireless communication systems that will be based on dynamic spectrum allocation (DSA) principle. This basic idea is the keystone of the Cognitive Radio (CR) concept, a technology that envisages a flexible access to unused or underutilized frequency spectrum from primary licensed networks and consequently solves the spectrum utilization problem.

The concept of the CR has been firstly proposed by Mitola J. III [3] and can be described as a software defined radio (SDR) based system that senses and is aware of its operational environment and can dynamically and autonomously adjust its radio operating parameters [4]. The key attributes of the CR are collaborative spectrum sensing (awareness of its surroundings) and adaptation ability of transmission features (TX frequency, technique of transmission, direction of transmission etc).

Spectrum agility and the adaptation process of the CR are shown in Fig. 1. Firstly, spectrum sensing and signal waveform determination functions are employed in order to find proper transmission resources, so called "spectrum holes". This function can be based on the real-time spectrum scanning and moreover, on the knowledge of the local environment (e.g. a regional frequency allocation database and statistics downloaded via cognitive pilot channel [5]). Next, the best course of action upon spectrum findings is taken. This includes a new allocation of resources and process initiation. Finally, the reconfigurable transceiver is adjusted accordingly. This whole process is dynamic and has to be updated regularly, according to the space-time spectrum availability and the actual transmission request (data rate, BER, latency, etc).

It is obvious, that the correct determination of spectrum holes and the ability to estimate the utilization is a very critical issue for DSA algorithms and hence, understanding the spectrum utilization becomes very important. Results of statistical measurements of the spectrum utilization could be possibly used and updated by CRs and measurement stations in particular geographical regions in order to facilitate finding of unused spectrum. The main objective of this paper is to present behavior of the radio spectrum utilization in the typical suburban environment. The measurement method, measurement equipment, data processing and major conclusions of the spectrum utilization analysis will be presented in following sections respectively.

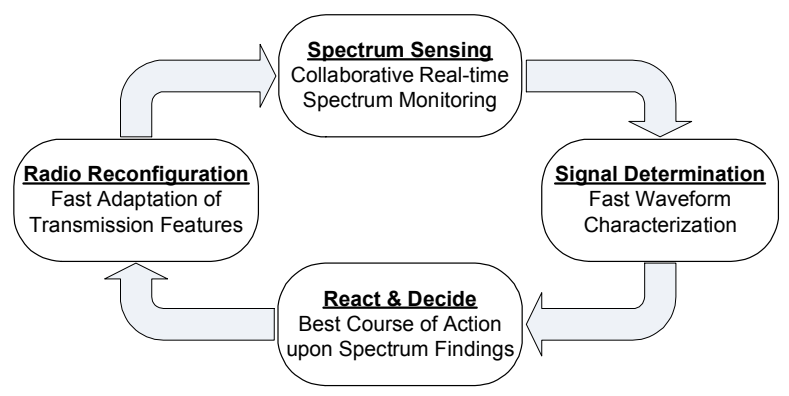

Fig. 1. Spectrum agility process of the CR: from spectrum sensing and intelligent decision to reconfiguration [7]. 


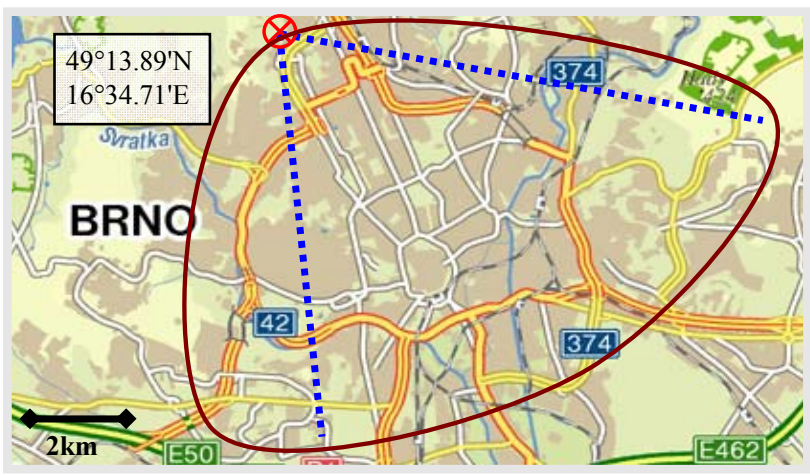

Fig. 2. Site of the measurement and the area of coverage.

\section{MEASUREMENT METHOD}

Spectrum utilization measurement has been carried out on the roof of the Department of Radioelectronics, which is located in the northern suburb of the town of Brno (medium industry town of 380,000 inhabitants), Czech Republic. The wideband antenna has been pointed towards the town in 3 directions day by day in order to cover the whole urban area. Vertical and horizontal polarization of the logarithmically periodic antenna has been considered. This measurement is different to those implemented in other studies (e.g. [1], [2]) since we focus on the urban spectrum utilization seen from the suburb area. As it will be shown later on, certain frequency bands in this area exhibit much lower utilization than expected and therefore, the frequency reuse by the CR can be even more extended. Measurements have been carried out during six subsequent days - 3 days using vertical polarization and 3 days with horizontal polarization. The overall result of this measurement is depicted in Fig. 3. In-depth utilization analysis of selected bands will be presented in Section IV.

\section{A. Measurement Equipment}

The signal captured by the antenna has been analyzed in spectrum analyzer Rohde\&Schwarz FSP and then loaded via GPIB/USB to PC using Matlab Instrument Control Toolbox. Matlab tool has been used for signal analysis of stored data. Radiation diagram of the log-periodic antenna is depicted in Fig. 4. It can be seen, that the directivity is relatively low and therefore, only three directions can be analyzed in order to cover the main part of the town. Correction factors due to the antenna gain, cable loss and connections have been considered as well.

\section{B. Data Processing}

The whole frequency band of $2.9 \mathrm{GHz}(100 \mathrm{MHz}-$ $3 \mathrm{GHz}$ ) has been divided into $14520-\mathrm{MHz}$ sub-bands. In order to achieve high resolution, each sub-band has been analyzed using resolution bandwidth of $3 \mathrm{kHz} .125$ samples have been stored for every single band, which in turn makes 18,125 samples per the whole analyzed bandwidth. 125 samples per band correspond to one sample every $160 \mathrm{kHz}$. Sweep time of 4 seconds has been set for every $20 \mathrm{MHz}$ sub-band and hence, the total scanning time of the $2.9 \mathrm{GHz}$ band results in 580 seconds. This measurement has been performed repeatedly for every antenna direction and both antenna polarization during 24 hours and over six days.

\section{SPECTRUM UTILIZATION ANALYSIS}

Figure 3 displays only rough information of the whole band of interest. In order to get more characteristic information about the spectrum utilization, we will zoom on selected licensed frequency bands and analyze them in a detail. Following frequency bands have been chosen: 380-385/390-395 MHz (TETRA), 410.2-412.8/420.2$422.8 \mathrm{MHz}$ (CDMA), 451.5-455.54/461.5-465.54 MHz (the first CDMA2000 in $450 \mathrm{MHz}$ ), 470-862 MHz (TV), 888-915/933-960 MHz (E-GSM 900), 1710-1785/1805$1880 \mathrm{MHz}$ (GSM 1800), 1920-1980/2110-2170 MHz (UMTS TDD) and 2400-2483.5 MHz (ISM).

The utilization of selected frequency bands has been defined by the duty cycle, which specifies the fraction of time the band is used. The duty cycle in considered frequency bands has been calculated as a ratio of number of samples $N_{\text {(P)threshold) }}$ with power level superior to the threshold value and the total number of samples $N_{\text {Total }}$.

$$
\text { Duty Cycle }=\frac{N_{\text {(P>threshold) }}}{N_{\text {Total }}}
$$

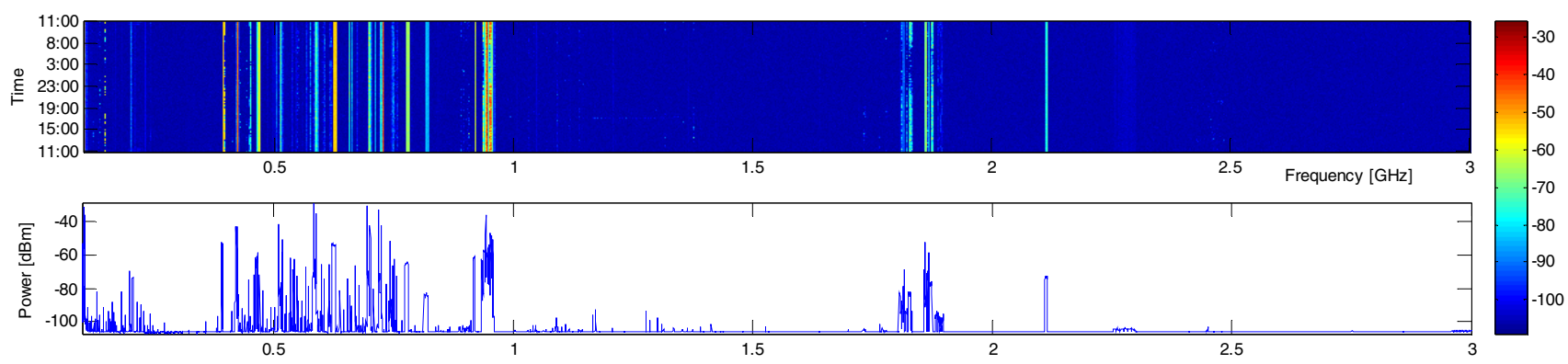

Fig. 3. Overall result of the temporal (upper plot) and average plot of the radio spectrum occupancy (colorbar in $\mathrm{dBm}$ ). 


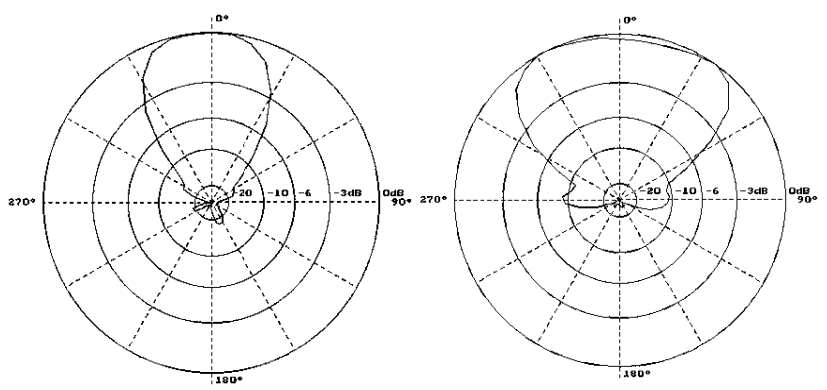

Fig. 4. Radiation diagram of the wideband log-periodic antenna in the vertical (left) and horizontal plane (right).

Determination of the power threshold is a critical issue. An excessive threshold may lead to overlooking potential activity within the band, but on the other hand, too low values may result in false alarm due to noise. In order to overcome this problem, various thresholds for different frequency bands have been defined, depending on the level of the background noise. The power threshold that determines the occupancy of a frequency sample has been set to $7 \mathrm{~dB}$ above the average value of the noise measured around the actual band. $7 \mathrm{~dB}$ has been chosen as the optimal value based on long term observation of the noise.

Following figures provide a detailed analysis of the utilization of selected bands. Each pair of figures presents occupied frequencies and the duty cycle respectively.

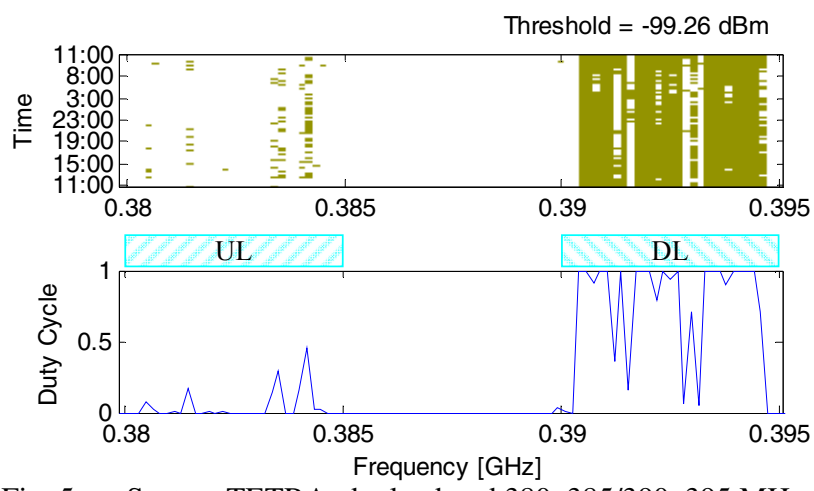

Fig. 5. System TETRA, duplex band 380-385/390-395 MHz.
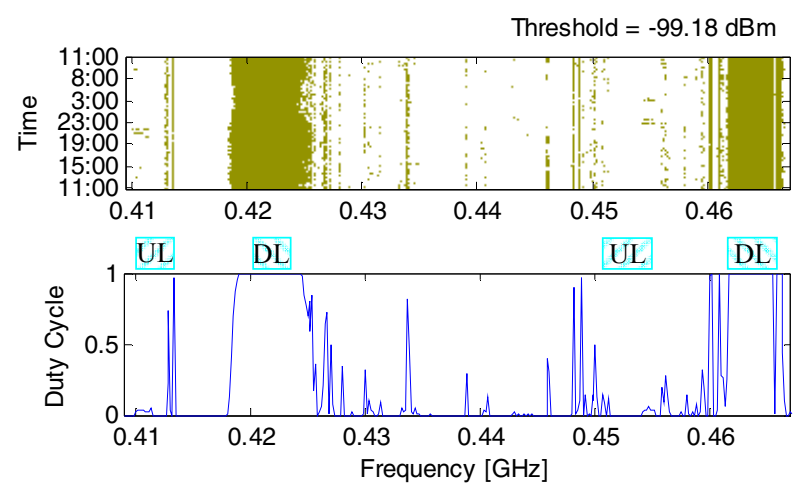

Fig. 6. Two different CDMA systems in duplex bands 410.2412.8/420.2-422.8 and 451.5-455.54/461.5-465.54 MHz.
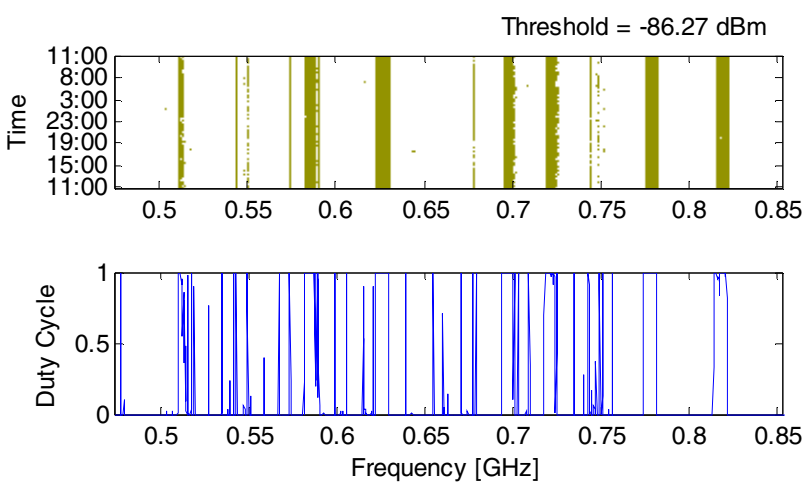

Fig. 7. TV channels 21 up to 69 in band of 470-862 MHz.
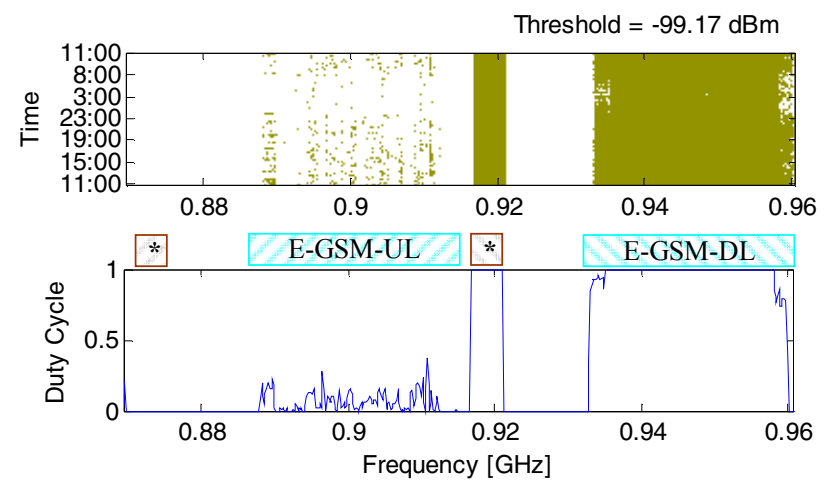

Fig. 8. E-GSM in band of 888-915/933-960 MHz and UMTS TDD in band of $872-876 / 917-921 \mathrm{MHz}$ (asterisk field*).

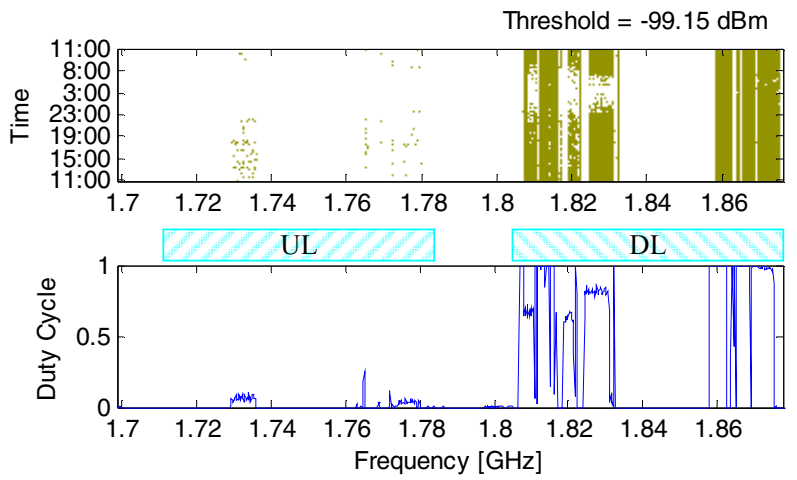

Fig. 9. GSM 1800 in band of $1710-1785 / 1805-1880 \mathrm{MHz}$.

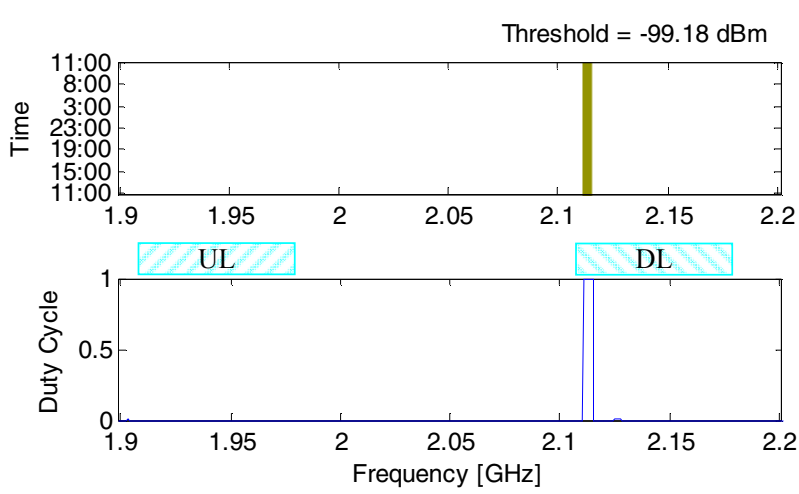

Fig. 10. UMTS TDD in band of 1920-1980/2110-2170 MHz. 

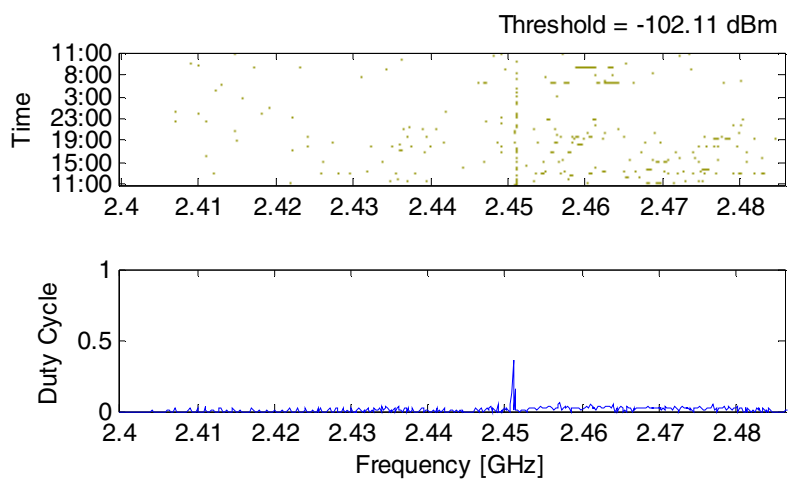

Fig. 11. ISM band of 2400-2483.5 MHz.

Frequency band below $380 \mathrm{MHz}$ exhibits the utilization of $5.38 \%$. This band is mainly allocated to broadcasting, aeronautical/maritime applications, fixed and mobile non civil communication and meteorological and radioastronomy applications.

Utilization of TETRA and GSM systems very much depends on the period of time (Fig. 5, Fig. 8-9). Moreover it has been observed, that downlink channels exhibit much higher activity than uplink channels. This is due to the fact, that the transmitting power of base stations is considerably higher than the power transmitted by individual mobile stations and moreover, the power attenuation due to the built-up area is much lower in case of base stations since they are situated on top of buildings.

Figures 6, 8 and 10 show performance of CDMA based systems (CDMA2000 EV-DO and UMTS TDD). Due to the nature of the wideband CDMA, only downlink signal has been observed. Uplink activity has not been detected since the energy of the signal is spread over a wide band in order to transmit lower power and therefore, such noiselike signals are hard to detect using the energy detection principle. Detection of these signals is generally based on the feature detection since the wideband signals have very distinct spectral correlation properties [6].

Figure 11 depicts relatively low utilization performance

TABLE I

SUMMARY OF THE SPECTRUM UTILIZATION

\begin{tabular}{|l|c|c|}
\hline System & Frequency [MHz] & Utilization [\%] \\
\hline TETRA & $380-385 / 390-395$ & 37.5 \\
\hline CDMA & $\begin{array}{c}410.2-412.8 / 420.2-422.8 \\
451.5-455.54 / 461.5-465.54\end{array}$ & $50.7 / 47.2$ \\
\hline TV & $470-862$ & 20.4 \\
\hline E-GSM & $888-915 / 933-960$ & 51.9 \\
\hline GSM 1800 & $1710-1785 / 1805-1880$ & 21.8 \\
\hline UMTS TDD & $1920-1980 / 2110-2170$ & 3.8 \\
\hline ISM & $2400-2483.5$ & 1.0 \\
\hline TOTAL & $\mathbf{1 0 0 - 3 0 0 0}$ & 6.96 \\
\hline
\end{tabular}

in the $2.4 \mathrm{GHz}$ ISM band. One explanation could be that most of wireless systems working in this band use highly directive antennas in order to cover small spots. Moreover, propagation of $2.4 \mathrm{GHz}$ frequencies in the urban area is very poor.

Utilization of TV bands presents a significant frequency reuse opportunity in our location. As seen in Fig. 7, many TV channels appear to be unused or are very weak. This is due to the fact that the TV channel allocation depends on the geographical location. Only ten out of 49 allocated channels were considered as occupied, which in turn results in $20.4 \%$ utilization of the whole band.

\section{CONCLUSION}

Utilization of the radio spectrum has been measured and selected licensed frequency bands have been analyzed in a detail. It has been shown that the total utilization of considered radio spectrum is lower than $6.96 \%$. These results demonstrate that there is a lot of underutilized space in the frequency spectrum, which could be used by CR technologies based on DSA. Our results reveal spectrum utilization in particular environment however, this work is still preliminary. It is obvious that more indepth studies are necessary to get more appropriate data, e.g. spectrum utilization analyzed in a statistical manner over much longer period and wide range of locations.

\section{ACKNOWLEDGEMENT}

This work was supported by the French Government program Doctorat en Cotutelle and by the Czech Grant Agency under grant no. 102/08/H027 and 102/07/1295.

\section{REFERENCES}

[1] S. D'Itri, M. McHenry, Dynamic spectrum access moves to the forefront. Defence Electronics, April 2008, p. S3 - S6.

[2] I. Habibul et al, "Spectrum survey in Singapore: occupancy measurements and analyses," in $3^{\text {rd }}$ Int. Conf. on Cognitive Radio Wireless Networks and Commun., Singapore, 2008.

[3] J. Mitola et al, Cognitive radios: making software radios more personal, IEEE Personal Commun., no. 4, Aug. 1999.

[4] F. K. Jondral, "From Maxwell's equations to cognitive radio," in $3^{\text {rd }}$ Int. Conference on Cognitive Radio Wireless Networks and Communications, Singapore, 2008.

[5] P. Houze, S. Ben Jemaa, P. Cordier, "Common pilot channel for network selection," VTC - Spring Conference, Melbourne, 2006.

[6] W. Gardner, W. Brown III, and C.-K. Chen, "Spectral correlation of modulated signals: Part II - digital modulation," IEEE Trans. Commun., vol. COM-35, no. 6, pp. 595-601, June 1987.

[7] J. B. Evans, "Intelligence in th Network," Keynote speech, Available:http://www.crowncom.org. [Accessed July 22, 08] 